\title{
Blood Platelet Count of Sucklings according to Arakawa's Reaction of Their Mothers.
}

rarst Report of the Peroxidase Reaction.

\author{
By \\ Masayuki Shindo. \\ (進 症正之) \\ (From the Department of Pediatrics, Faculty of Medicine, \\ Tohoku Imperial University, Sendai. \\ Director: Prof. A. Sato.)
}

\section{Introduction.}

Our Department has already shown hematologically that lactating women secreting milk negative to Arakawa's reaction are in, or at least are inclined to, a state of avitaminosis $\mathrm{B}^{*}$.

Chiba and $\mathrm{Abe},{ }^{1)} \mathrm{Chiba},{ }^{2)}$ and $\mathrm{Suzuki}^{3) 4}$ have shown the above mentioned conclusion on the basis of the prolonged short-timed peroxidase reaction of blood leucocytes, $\mathrm{Kimura}{ }^{5) 6}$ on the basis of the increased blood platelet count, Shira is $\mathrm{hi}^{7)}$ on the basis of increased reticulocyte count. Ko k ubo ${ }^{8)}$ reported that leucocytosis resulting from neutrophilia as well as from lymphocytosis and nuclear shift to the left were found in mothers secreting Arakawa-negative milk. Shiraish ${ }^{9)}$ concluded that a very close relation existed between a remarkable prolongation of the short-timed peroxidase reaction of leucocytes in infants nursed with Arakawa-negative milk and a large

\footnotetext{
* $B_{1}$ is meant throughout the article.

1) M. Chiba and J. A be, Tohoku J. Exp. Med, 1932, 19, 479.

2) M. Chiba, Tohoku J. Exp. Med., 1932, 19, 486.

3) T. S z z u ki, Tohoku J. Exp. Med., 1934, 23, 23.

4) T. Suzuki, Tohoku J. Exp, Med., 1935, 25, 186.

5) J. Kim u r a, Tohoku I. Exp. Med., 1934, 23, 494.

6) J. Kimura, Tohoku J. Exp. Med., 1935, 27, 360.

7) Sh. Shiraishi, Tohokn J. Exp. Med., 1936, 28, 44.

8) Y. Koku b o, Tohoku J. Exp., Med., 1936, 29, 371.

9) Sh. Shiraishi, Tohoku J. Exp. Med., 1937, 31, 268.
} 
reticulocyte count in their mothers. Except for six reports 1) 2) 3) 4) 9) 10) concerning the short-timed peroxidase reaction of leucocytes of infants, three reports of blood finding from the side of babies have been published. Ohta and Suzuki ${ }^{10}$ stated that a prolongation of the shortest peroxidase stain-time of blood leucocytes, a sign of avitaminosis $B$, would occur in infants with a high blood platelet count, another sign of avitaminosis B. Shiraishi and $I^{11)}$ examined the blood picture in 15 cases of infantile preberiberi in comparison with those of 5 cases of infantile beriberi and of 3 cases of B-avitaminotic dyspepsia, and observed a high blood platelet count in these cases. Further, by investigating 237 cases of blood platelets in infants $\mathrm{I}^{12)}$ obtained the result that in cases of sucklings fed on milk negative to Arakaw a's reaction, anemia and thrombocytosis were seen, and that blood platelet counts were larger according as A rak a wa's reaction became weaker.

In the present paper I desire to report on the relation between Arakawa's reaction of mothers and blood platelet count of infants, and further on the difference between the cases in which both infants and their mothers had not been taking any vitamin $B$ preparation, and those in which infants and/or their mothers had been taking a vitamin B preparation.

\section{Method of Experiment.}

1. Materials. Out- and in-patients of our Pediatric Department during the time from summer of 1934 to spring of 1936.

2. Blood taking. Blood was obtained from an ear lobe and care was taken to avoid disturbance of babies as much as possible.

3. Method. Fonio's method with a slight modification was used. A couple of films were fixed with methyl-alcohol three minutes duration and then stained with Gi ems a's stain (2 drops of the stain in 1 c.c. of distilled water) as long as 12 hours, so that platelets were stained quite distinctly.

4. A rakaw a's reaction. All the cases were divided into 3 groups according to the intensity of Ara ka wa's reaction.

a. The normally or strongly Arakawa-positive group: Arakaw a's reaction of milk from both breasts was normally or strongly positive in one minute.

b. Group with intermediate A ra kawa's reaction: A rakawa's reaction was chemically positive but clinically negative.

10) F. Ohta and T. S u zuki, Tohoku J. Exp. Med., 1987, 31, 247.

11) Sh. Shira ishi and M. Shindo, Tohoku J. Exp. Med., 1938, 32, 470.

12) M. Shind o, Tohoku J. Exp. Med, 1939, 36, 258. 
c. The Arakawa-negative group: Arakawa's reaction of milk from both breast was completely or almost completely negative after five minutes.

The different courses of Arakawa's reaction during 5 minutes were shown by special signs (Cf. Table 1) for simplification.

\section{Table 1.}

Table of signs for showing different A rakawa's reaction.

$\begin{array}{lllll}1^{\prime} & 2^{\prime} & 3^{\prime} & 4^{\prime} & 5^{\prime}\end{array}$

1. $1(H)$ stands for $H$ H 4 H H

2. $1(H)=H+H+H+H$

3. $2(H)$, $H$ H H H

4. $3(H) \rightarrow$ H H H H H

5. $4(H)=H+H H$

6. $5(H)$ \# H H H H

7. $1(+) \quad+H$ H H H

8. $2(+) \quad n+H+H H$

9. $3(+) \quad n+H+H$

10. $4(+), \quad+H+H+H$

11. $5(+)=++H$ H H

12. $6(+) "+++H$

13. $7(+) \pi++H+H$

14. $8(+) \quad+t+H$

15. $9(+)$ n $+++H+$

16. $10(+)$ " +++++

17. $11(+)$ n +++++

18. $1( \pm) \quad$ $\quad \pm+H$ H H

19. $2( \pm) \quad \pi \quad \pm+H+\#$

20. $3( \pm), \pm+H+H$

21. $4( \pm)$ n $\pm++H+H$

22. $5( \pm) \quad \pm++H+H$

23. 6(土), \pm++++

24. $7( \pm)$ " \pm++++ $\begin{array}{llllll} & 2^{\prime} & 3^{\prime} & 4^{\prime} & 5^{\prime}\end{array}$

25. $8( \pm)$ stands for $\pm \pm+H+H$

26. $9( \pm), n \pm \pm+H+$

27. $10( \pm)$ " $\pm \pm++H$

28. $11( \pm), \quad \pm \pm+++$

29. $12( \pm) \quad n \quad \pm \pm \pm+H$

30. $13( \pm) \quad n \quad \pm \pm \pm++$

31. 14(土),$\quad \pm \pm \pm \pm+$

32. $15( \pm), \quad \pm \pm \pm \pm \pm$

33. $1(-) \quad n \quad- \pm+H+$ H

34. $2(-)$ n $- \pm++H$

35. $3(-) \quad$ - $\pm++H$

36. $4(-) \quad n- \pm+++$

37. $5(-), \quad- \pm \pm++$

38. $6(-)$ n - \pm \pm+

39. $7(-)$ "

40. $8(-) \quad n- \pm \pm \pm \pm$

41. $9(-) \quad, \quad-- \pm+H$

42. $10(-) \quad n- \pm++$

43. 11(-) n - - 士 士 +

44. $12(-) \quad n \quad-\quad \pm \pm \pm$

45. $13(-) \quad n--\cdots \pm+$

46. $14(-), \quad--- \pm \pm$

47. $15(-) \quad$ " ---- \pm

48. $16(-)$ -

Explanation to the table:-

Take, for instance, the sign: $2(H)$. This stands for A rakawa's reaction with the course $(H) 1^{\prime}(H) 2^{\prime}(H) 3^{\prime}(H) 4^{\prime}(\#) 5^{\prime}$. The sign does not express any prompt result of the reaction, so the prompt reaction of the sign: $2(H)$ may be $(-) 0^{\prime},( \pm) 0^{\prime}$, $(+) 0^{\prime}$ or even $(H) 0^{\prime}$, but this will not matter much, as the result of the reaction in one minute is the most important.

\section{Results.}

The cases investigated were 345 in all, and 96 cases among them had already been taking vitamin $B$ preparations. All the cases were divided into 3 groups according to the intensity of A rakaw a's reaction. Normally Arakawa-positive cases were shown in Tables 2 and 3 , cases with intermediate Arakawa's reaction in Tables 4 and 5 , and completely or almost completely Arakawa-negative cases in Tables 6 
TABLE 2.

Normally Arakawa-positive eases in which infants and their mothers had not been taking vitamin $B$ preparation.

\begin{tabular}{|c|c|c|c|c|c|c|c|c|}
\hline \multirow{2}{*}{ No. } & \multirow{2}{*}{ Name } & \multirow{2}{*}{$\operatorname{Sex}$} & \multirow{2}{*}{$\begin{array}{c}\text { Age } \\
y=\text { years } \\
\mathrm{m}=\text { months } \\
\mathrm{d}=\text { days }\end{array}$} & \multirow{2}{*}{ Diagnosis* } & \multicolumn{2}{|c|}{$\begin{array}{c}\text { Arakawa's } \\
\text { reaction }\end{array}$} & \multirow{2}{*}{$\begin{array}{c}\text { Red cell } \\
\text { count } \\
\text { per cmm. } \\
\text { (thousands) }\end{array}$} & \multirow{2}{*}{$\begin{array}{c}\text { Blood plate } \\
\text { let count } \\
\text { per cmm. } \\
\text { (thousands) }\end{array}$} \\
\hline & & & & & Right & Left & & \\
\hline 1 & E.A. & 우 & $91 d$ & $\mathbf{P}$ & $1(+4)$ & 1 (ffit) & 5,620 & 290 \\
\hline 2 & K.M. & 实 & $11 \mathrm{~m}$ & $D$ & 1 (it) & $1(H)$ & 4,650 & 410 \\
\hline 3 & S. A. & 우 & $8 \mathrm{~m}$ & $\mathrm{D}$ & $1(H)$ & $2(+t)$ & 5,800 & 271 \\
\hline 4 & K.O. & 우 & $1 \mathrm{y} 1 \mathrm{~m}$ & $\mathbf{R}$ & $1(H)$ & 1 (地) & 5,100 & 280 \\
\hline 5 & K.I. & 우 & $5 \mathrm{~m}$ & $\mathrm{He}$ & $1(+)$ & $I(H)$ & 5,950 & 310 \\
\hline 6 & K.H. & $\hat{\beta}$ & $4 m$ & $\mathrm{II}$ & $1(+t)$ & $1(+)$ & 4,800 & 293 \\
\hline 7 & K.S. & ث & $10 \mathrm{~m}$ & $\mathrm{He}$ & $1(+t)$ & $1(+1)$ & 5,310 & 460 \\
\hline 8 & J.J. & 우 & $3 \mathrm{~m}$ & $\mathrm{H}$ & $1(4)$ & $1(t+)$ & 4,000 & 337 \\
\hline 9 & Y.T. & $\delta$ & $9 \mathrm{~m}$ & $\mathbf{R}$ & $1(+t)$ & $1(t)$ & 4,950 & 460 \\
\hline 10 & R.A. & 今 & $5 \mathrm{~m}$ & D & $1(+t)$ & $1(H)$ & 5,550 & 347 \\
\hline 11 & K.M. & 今 & $11 \mathrm{~m}$ & $\mathbf{R}$ & $1(+4)$ & $1(+t)$ & 5,930 & 350 \\
\hline 12 & H.U. & $\widehat{\delta}$ & $10 \mathrm{~m}$ & $\mathrm{Br}$ & $2(+t)$ & $2(+t)$ & 4,350 & 249 \\
\hline 13 & T.S. & 今 & $5 \mathrm{~m}$ & $\mathrm{He}$ & $1(H)$ & $1(t+t)$ & 4,270 & 320 \\
\hline 14 & E.T. & 우 & $7 \mathrm{~m}$ & I & $1(t+1)$ & $1(\mathrm{Ht})$ & 3,850 & 159 \\
\hline 15 & M.S. & 웅 & $9 \mathrm{~m}$ & $\mathbf{A}$ & $1(H)$ & $1(H)$ & 5,370 & 234 \\
\hline 16 & J.S. & $\delta$ & $5 \mathrm{~m}$ & $\mathbf{R}$ & $1(++)$ & $1(++)$ & 5,270 & 373 \\
\hline 17 & Y.N. & 우 & $6 \mathrm{~m}$ & I & $1(t+4)$ & $1(+4)$ & 4,730 & 230 \\
\hline 18 & Y.I. & 우 & $38 \mathrm{~d}$ & $\mathbf{A}$ & $1(t+)$ & $1(t+1)$ & 6,950 & 341 \\
\hline
\end{tabular}

Abbreviation used in Tables 2, 3, 4, 5, 6 and 7 .

\begin{tabular}{|c|c|c|}
\hline$A=$ Atrophy, & An $=$ Angina, & $\begin{aligned} \mathbf{B}= & \text { B-avitaminotic } \\
& \text { dyspepsia }\end{aligned}$ \\
\hline $\begin{aligned} \mathrm{Ba} & =\mathrm{B} \text {-avitaminosis } \\
\mathrm{C} & =\text { Chondrodystrophia }\end{aligned}$ & $\begin{array}{l}\mathrm{Bp}=\text { Bronchopneumonia }, \\
\mathrm{Cn}=\text { Constipation. }\end{array}$ & $\mathrm{Br}=$ Bronehitis \\
\hline $\begin{aligned} \text { De } & =\text { Decomposition, } \\
\mathrm{H} & =\text { Hypotrophia }\end{aligned}$ & $\begin{aligned} \mathbf{E} & =\text { Eczema, } \\
\text { He } & =\text { Hydrocephalus }\end{aligned}$ & $\begin{array}{l}\mathrm{Ep}=\text { Empyema pleurae, } \\
\mathrm{He}=\text { Healthy }\end{array}$ \\
\hline $\mathrm{Hi}=$ Hernia inguinalis, & $\begin{aligned} \mathrm{Hm}= & \text { Heine-Medin's } \\
& \text { disease, }\end{aligned}$ & \\
\hline $\begin{aligned} \mathrm{Ht} & =\text { Hydrocele testis } \\
\mathrm{I} & =\text { Influenza, }\end{aligned}$ & $\begin{aligned} \text { Hu} & =\text { Hernia umbilicalis, } \\
\mathrm{Ib} & =\text { Infantile beriberi, }\end{aligned}$ & $\begin{aligned} H y & =\text { Hypogalaktia } \\
\text { Ie } & =\text { Ileocolitis }\end{aligned}$ \\
\hline $\mathrm{Id}=\mathrm{Idiocy}$ & Im $=$ Impetigo & Ip $=$ Infantile preberiberi \\
\hline $\begin{array}{l}\text { Le = Lues congenita, } \\
\mathrm{Pb}=\text { Pharyngobronchitis, }\end{array}$ & $\begin{aligned} N & =\text { Nephritis } \\
\mathrm{Pc} & =\text { Pyelocytitis }\end{aligned}$ & $\begin{aligned} \mathbf{P} & =\text { Pertussia } \\
\mathrm{Pe} & =\text { Parotitis epidemica }\end{aligned}$ \\
\hline Ps = Pylorospasmus, & $\begin{aligned} P_{\mathrm{sh}}= & \text { Pseudohemophilia } \\
& \text { hepatica, }\end{aligned}$ & \\
\hline $\mathrm{R}=$ Rhinopharyngitis, & $\begin{array}{c}\text { hepatica, } \\
\mathrm{Si}=\text { Strophulus } \\
\text { infantum, }\end{array}$ & St $=$ Stomatitis, \\
\hline $\mathrm{n}=$ Wry-neck, & $P=$ Suspicion of & \\
\hline
\end{tabular}

and 7 respectively. Fach group was divided again into two subgroups:-The cases in which both infants and their mothers had not been taking any vitamin B preparation (Cf. Tables 2, 4 and 6) and the cases in which infants and/or their mothers had been taking a vitamin. B preparation (Cf. Tables 3, 5, and 7). 
TABLE 3.

Normally Arakawa-positive cases in which infants and/or their mothers had been taking vitamin $B$.

\begin{tabular}{|c|c|c|c|c|c|c|c|c|c|c|}
\hline \multirow{2}{*}{$\begin{array}{c}\dot{0} \\
\ddot{4}\end{array}$} & \multirow{2}{*}{ Name } & \multirow{2}{*}{$\begin{array}{l}x \\
0 \\
0 \\
0\end{array}$} & \multirow{2}{*}{$\begin{aligned} & \text { Age } \\
\mathrm{y}= & =\text { years } \\
\mathrm{m} & =\text { months } \\
\mathrm{d} & =\text { days }\end{aligned}$} & \multirow{2}{*}{ 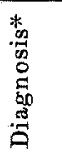 } & \multicolumn{2}{|c|}{$\begin{array}{l}\text { Arakawa's } \\
\text { reaction }\end{array}$} & \multirow{2}{*}{$\begin{array}{l}\text { Red cell } \\
\text { count } \\
\text { per cmm. } \\
\text { (thou- } \\
\text { sands) }\end{array}$} & \multirow{2}{*}{$\begin{array}{c}\text { Blood } \\
\text { platelet } \\
\text { count per } \\
\text { c.m.m. } \\
\text { (thou- } \\
\text { sands) }\end{array}$} & \multicolumn{2}{|c|}{$\begin{array}{l}\text { Administra- } \\
\text { tion of } \\
\text { vitamin } B \text { to }\end{array}$} \\
\hline & & & & & Right & Left & & & Mother & Infant \\
\hline 1 & I. $\mathrm{H}$. & $\hat{0}$ & $58 d$ & $\mathrm{He}$ & $1(+\#)$ & $1(+4)$ & 4,700 & 270 & $(+)$ & $(+)$ \\
\hline 2 & T.K. & 今 & $9 \mathrm{~m}$ & I & $1(+4)$ & $1(H)$ & 5,550 & 271 & & \\
\hline 3 & S.I. & $\boldsymbol{\delta}$ & $1 \mathrm{~g} 4 \mathrm{~m}$ & D & $1(H)$ & $2(t)$ & 6,500 & 200 & $(-$ & $(+1)$ \\
\hline 4 & $\mathrm{E}, \mathrm{M}$. & $\delta$ & $4 \mathrm{~m}$ & $\mathrm{H}$ & $1(\#)$ & $1(t)$ & 4,570 & 230 & $(+)$ & $(+)$ \\
\hline 5 & Y.K. & 우 & $3 \mathrm{~m}$ & Wn & $1(H)$ & $2(t)$ & 4,440 & 242 & & $(+)$ \\
\hline
\end{tabular}

* Cf. Foot note to Table 2.

1. Distribution of blood platelets and red cell count according to the intensity of A rakawa's reaction (Cf. Table 8).

From Table 8 it is seen that there was no difference of the average count (about 5,100 thousand cells) of erythrocytes between the normally Arakawa-positive group and the cases with intermediate A rakaw a's reaction, but in the Arakawa-negative group red cell count showed a decrease, being 4,930 thousand cells on an average. Infants fed on milk negative to Arakawa's reaction ware thus in a state of anemia, though the degree was slight.

As to blood platelet count, it was 301,384 and 435 thousands according to the intensity of Arakawa's reaction. In the normally Arakawa-positive group it was within the normal value as will be mentioned in the next paragraph, while a considerable increase of blood platelet count was seen in the Arakawa-negative group.

2 . Distribution of red cell count and blood platelet count according to the intensity of Arakawa's reaction and according as a vitamin B preparation had been taken or not (Cf. Table 9).

Erythrocyte count in the Arakawa-negative group was, as stated above, smaller than the other two groups. But the influence of vita$\min \mathrm{B}$ will be seen in each of the three groups. An inclination, though very slight, to an increase of the count will be seen in the subgroup. with vitamin $B$.

In each group of A rakawa's reaction, thrombocyte count showed a decrease in the subgroup with vitamin $B$. The difference between the two subgroups in each group was 74,000,46,000 and 79,000according to the intensity of Arakawa's reaction respectively. And the 


\section{TABLE 4.}

Cases with intermediate A rakawa's reaction in which infants and their mothers had not been taking vitamin $B$ preparation.

\begin{tabular}{|c|c|c|c|c|c|c|c|c|}
\hline \multirow{2}{*}{ No. } & \multirow{2}{*}{ Name } & \multirow{2}{*}{ Sex } & \multirow{2}{*}{$\begin{aligned} & \text { Age } \\
\mathrm{y} & =\text { years } \\
\mathrm{m} & =\text { months } \\
\mathrm{d} & =\text { days }\end{aligned}$} & \multirow{2}{*}{ Diagnosiş } & \multicolumn{2}{|c|}{$\begin{array}{c}\text { Arakawa's } \\
\text { reaction }\end{array}$} & \multirow{2}{*}{$\begin{array}{c}\text { Red cell } \\
\text { eount } \\
\text { per cmm. } \\
\text { (thousands) }\end{array}$} & \multirow{2}{*}{$\begin{array}{c}\text { Blood } \\
\text { platelet } \\
\text { count per } \\
\text { cmm. } \\
\text { (thousands) }\end{array}$} \\
\hline & & & & & Right & Left & & \\
\hline 1 & M.H. & 古 & $47 \mathrm{~d}$ & Ip & $2( \pm)$ & $2( \pm)$ & 5,810 & 530 \\
\hline 2 & Y.S. & o & $5 \mathrm{~m}$ & $\mathrm{R}$ & $1(+\#)$ & $6(-2)$ & 4,750 & 304 \\
\hline 3 & Y.A. & 9 & $4 \mathrm{~m}$ & $\mathrm{D}$ & $16(-)$ & $10( \pm)$ & $4,470^{\circ}$ & 538 \\
\hline 4 & M.N. & $\delta$ & $8 \mathrm{~m}$ & $\mathrm{R}$ & $1(H)$ & $8(-)$ & 6,000 & 282 \\
\hline 5 & Y.Y. & 范 & $5 \mathrm{~m}$ & I & $10( \pm)$ & $1(+)$ & 4,800 & 370 \\
\hline 6 & S. N. & 今 & $6 \mathrm{~m}$ & $\mathrm{Si}$ & $4( \pm)$ & $3( \pm)$ & 5,350 & 327 \\
\hline 7 & A.T. & 우 & $9 \mathrm{~m}$ & I & $8(-)$ & $|15(-)|$ & 5,880 & 380 \\
\hline 8 & K.o. & \& & $2 \mathrm{~m}$ & $\mathbf{R}$ & $10(-)$ & $1(+)$ & 4,180 & 375 \\
\hline 9. & K.H. & $\delta$ & $11 \mathrm{~m}$ & $\mathrm{D}$ & $11(-)$ & $1(+1)$ & 5,860 & 430 \\
\hline 10 & R.T & 古 & $9 \mathrm{~m}$ & $\mathrm{Br}$ & $10( \pm)$ & $5(+)$ & 5,000 & 420 \\
\hline 11 & Y.K. & 1 & $8 \mathrm{~m}$ & $\mathrm{D}$ & $8(-)$ & $1(+)$ & 4,910 & 270 \\
\hline 12 & $\mathrm{~S}, \mathrm{~N}$ & $\delta$ & $3 \mathrm{~m}$ & $\bar{R}$ & $*$ & & 4,870 & 420 \\
\hline 13 & Y.W. & ฬิ & $36 d$ & $\mathrm{E}$ & $13( \pm)$ & $1(+)$ & 4,370 & 374 \\
\hline 14 & s.m. & ส & $3 \mathrm{~m}$ & $\mathrm{D}$ & $3( \pm)$ & $3( \pm)$ & 5,050 & 810 \\
\hline 15 & S. M. & ह & $5 \mathrm{~m}$ & $R+D$ & $13( \pm)$ & $1(+)$ & 4,670 & 313 \\
\hline 16 & $\mathrm{~K} . \mathrm{A}$. & 字 & $7 \mathrm{~m}$ & $\mathrm{Lc}$ & $10( \pm)$ & $11(t)$ & 3,750 & $520 \cdots$ \\
\hline 17 & $\mathrm{~T}, \mathrm{~T}$ & 우 & $4 \mathrm{~m}$ & $\mathrm{~B}$ & $11( \pm)$ & $9(-)$ & 5,110 & 390 \\
\hline 18 & J.S. & $\delta$ & $36 \mathrm{~d}$ & Psh & $10(-)$ & $2(-)$ & 2,030 & 278 \\
\hline 19 & S. N. & s & $9 \mathrm{~m}$ & $\mathrm{D}$ & $14( \pm)$ & $15( \pm)$ & 4,430 & 425 \\
\hline 20 & M.K. & 今 & $9 \mathrm{~m}$ & Lo & $1(t+)$ & $1(+)$ & 5,740 & 411 \\
\hline 21 & T.S. & 9 & $9 \mathrm{~m}$ & $\mathrm{D}$ & $1( \pm)$ & $16(-)$ & 5,750 & 218 \\
\hline 22 & M.W. & 우 & $12 \mathrm{~m}$ & $\mathbf{H}$ & $11(-)$ & $13(-)$ & 4,600 & 331 \\
\hline 23 & Y.K. & 8 & $37 \mathrm{~d}$ & $\mathbf{E}$ & $5( \pm)$ & $5( \pm)$ & 3,930 & 266 \\
\hline 24 & S. I. & s & $1 \mathrm{y} 1 \mathrm{~m}$ & $\mathbf{R}$ & $2(-5)$ & $3( \pm)$ & 4,500 & 290 \\
\hline 25 & E.T. & $\delta$ & $7 \mathrm{~m}$ & $\mathbf{R}$ & $5(+)$ & $1(+4)$ & 4,830 & 850 \\
\hline 26 & Y.X. & $\delta$ & $9 \mathrm{~m}$ & $D+A n$ & $11( \pm)$ & $1( \pm)$ & 5,280 & 270 \\
\hline 27 & K.T. & $\theta$ & $9 \mathrm{~m}$ & $\mathbf{P}$ & $1(t+4)$ & $1( \pm)$ & 4,990 & 278 \\
\hline 28 & H.M. & 우 & $35 \mathrm{~d}$ & $\mathrm{Hy}$ & $8( \pm)$ & $111-b$ & 4,580 & 254 \\
\hline 29 & K.K. & 实 & $10 \mathrm{~m}$ & $\mathbf{H m}$ & $1(+4)$ & $3(-5)$ & 5,560 & 311 \\
\hline 30 & H.K. & 웅 & $12 \mathrm{~m}$ & $\mathrm{Bp}$ & * & $*$ & 4,110 & 200 \\
\hline 31 & K.T. & 令 & $4 \mathrm{~m}$ & I & $2(-)$ & $* *$ & 5,150 & 311 \\
\hline 32 & $\mathrm{~K} . \mathrm{S}$ & 우 & $6 \mathrm{~m}$ & $\mathbf{D}+\mathbf{A}$ & $10( \pm)$ & $|15(-)|$ & 4,870 & 380 \\
\hline 33 & S. K. & $\widehat{\delta}$ & $35 \mathrm{~d}$ & Ip & $11(-)$ & $11(-)$ & 4,100 & 553 \\
\hline 34 & S. Y. & $\delta$ & $6 \mathrm{~m}$ & $\mathrm{D}$ & $14(-)$ & $9( \pm)$ & 5,440 & 370 \\
\hline 35 & K.S. & $\hat{\sigma}$ & $8 \mathrm{~m}$ & C & Fits & 年 & 4,470 & 431 \\
\hline 36 & S.S. & s & $2 \mathrm{~m}$ & B & $11(-)$ & $16(-)$ & 4,600 & 511 \\
\hline 37 & Y.T. & 우 & $10 \mathrm{M}$ & I & $6(+)$ & $5(-)$ & 4,750 & 680 \\
\hline 38 & M.F. & 오 & $9 \mathrm{~m}$ & Ip & $6(-)$ & $16(-)$ & 4,530 & 472 \\
\hline 39 & E.T. & 우 & $52 \mathrm{~d}$ & $B$ & $15( \pm)$ & $7( \pm)$ & 6,180 & 721 \\
\hline 40 & K. G. & $\hat{0}$ & $1 y$ & B & $6( \pm)$ & $6( \pm)$ & 5,240 & 688 \\
\hline 41 & J.T. & 就 & $4 \mathrm{~m}$ & B & $4(-)$ & $4(-)$ & 4,300 & 637 \\
\hline 42 & A.T. & के & $9 \mathrm{~m}$ & $\mathrm{Hm}$ & $1(t+)$ & $1+3$ & 5,190 & 330 \\
\hline 43 & M.A. & 우 & $5 \mathrm{~m}$ & $\mathrm{D}$ & $13(-)$ & $13(-)$ & 7,250 & 400 \\
\hline 44 & T.K. & o & $3 \mathrm{~m}$ & $\mathrm{D}+\mathrm{E}$ & 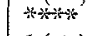 & $14(-)$ & 4,050 & 365 \\
\hline 45 & H.Y. & 8 & $1 \mathrm{~g} 2 \mathrm{~m}$ & $\mathbb{D}$ & $1(t)$ & $6( \pm)$ & 5,030 & 313 \\
\hline 46 & K.S. & $\delta$ & $1 \mathrm{y} 4 \mathrm{~m}$ & $D+D c$ & $16(-)$ & $6(-)$ & 5,600 & 480 \\
\hline 47 & T.S. & $\delta$ & $11 \mathrm{~m}$ & $\mathrm{D}$ & $5( \pm)$ & $4(-)$ & 5,200 & 460 \\
\hline 48 & Y.S. & 7 & $1 y 3 \mathrm{~m}$ & Ic & $12( \pm)$ & $12( \pm)$ & 4,800 & 230 \\
\hline 49 & K.T. & $\hat{\delta}$ & $5 \mathrm{~m}$ & $\mathbf{P}$ & $2( \pm)$ & $2( \pm)$ & 6,200 & 420 \\
\hline 50 & S. I. & $\hat{\delta}$ & $1 y 4 \mathrm{~m}$ & D & $2( \pm)$ & $1(+)$ & 6,500 & 222 \\
\hline
\end{tabular}


Table 4. Continued.

\begin{tabular}{|c|c|c|c|c|c|c|c|c|}
\hline \multirow{2}{*}{ No. } & \multirow{2}{*}{ Name } & \multirow{2}{*}{ Sex } & \multirow{2}{*}{$\begin{array}{c}\text { Age } \\
\mathrm{y}=\text { years } \\
\mathrm{m}=\text { months } \\
\mathrm{d}=\text { days }\end{array}$} & \multirow{2}{*}{ Diagnosis } & \multicolumn{2}{|c|}{$\begin{array}{c}\text { Arakawa's } \\
\text { reaction }\end{array}$} & \multirow{2}{*}{$\begin{array}{c}\text { Red cell } \\
\text { count } \\
\text { per cmm. } \\
\text { (thousands) }\end{array}$} & \multirow{2}{*}{$\begin{array}{c}\text { Blood } \\
\text { platelet } \\
\text { count per } \\
\text { cmm. } \\
\text { (thousands) }\end{array}$} \\
\hline & & & & & Right & $I_{s} \in f t$ & & \\
\hline 51 & H.S. & 令 & $71 \mathrm{~d}$ & D & $1(+)$ & $5( \pm)$ & 4,300 & 322 \\
\hline 52 & K.I. & 우 & $10 \mathrm{~m}$ & $\mathrm{Ep}$ & $5( \pm)$ & $6( \pm)$ & 6,750 & 540 \\
\hline 53 & Y.I. & 우 & $6 \mathrm{~m}$ & $\mathrm{Bp}$ & $11( \pm)$ & $4(+)$ & 6,900 & 420 \\
\hline 54 & $\mathrm{~K} . \mathrm{T}$. & 우 & $8 \mathrm{~m}$ & $D+A$ & $7( \pm)$ & $6(-)$ & 6,950 & 900 \\
\hline 55 & K.S. & 운 & $1 \mathrm{y} 1 \mathrm{~m}$ & $\mathbf{N}$ & $9(-)$ & $9(-)$ & 4,400 & 423 \\
\hline 56 & J. 0 . & $\delta$ & 31 d & Le & $13( \pm)$ & $14(-5)$ & 5,630 & 440 \\
\hline 57 & T.K. & ๘ & $9 \mathrm{~m}$ & I & $7(+)$ & $7(+)$ & 7,850 & 780 \\
\hline 58 & K.S. & 우 & $6 \mathrm{~m}$ & He & $4(+)$ & $1(+)$ & 4,250 & 215 \\
\hline 59 & S. T. & $\delta$ & $6 \mathrm{~m}$ & $\mathrm{Si}$ & $6(+)$ & $11( \pm)$ & 5,330 & 540 \\
\hline 60 & H.E. & $\delta$ & $12 \mathrm{~m}$ & B & $1(+)$ & $1(+)$ & 6,100 & 430 \\
\hline 61 & K.N. & 우 & $8 \mathrm{~m}$ & $\mathbf{R}$ & $3(+)$ & $7( \pm)$ & 4,970 & 200 \\
\hline 62 & M.K. & 苦 & $6 \mathrm{~m}$ & $\mathrm{D}$ & $1( \pm)$ & $1(H)$ & 5,970 & 401 \\
\hline 63 & Y.M. & $\delta$ & $1 \mathrm{y} 6 \mathrm{~m}$ & $A+D$ & $12(-)$ & $11( \pm)$ & 6,400 & 360 \\
\hline 64 & T.K. & 우 & $8 \mathrm{~m}$ & 0 & $1(+)$ & $1(+)$ & 6,250 & 320 \\
\hline 65 & K.K. & $\delta$ & $1 \mathrm{~g} 1 \mathrm{~m}$ & D & $1(-)$ & $6(+)$ & 7,350 & 630 \\
\hline 66 & J. K. & $\delta$ & $45 \mathrm{~d}$ & $\mathrm{D}$ & $5( \pm)$ & $1(H)$ & 4,430 & 250 \\
\hline 67 & K.I. & 우 & $75 \mathrm{~d}$ & $\mathrm{Ba}$ & $2(+)$ & $2(+)$ & 4,200 & 515 \\
\hline 68 & T.K. & 우 & $4 \mathrm{~m}$ & $\mathbf{R}$ & $1(H)$ & $4(+)$ & 5,700 & 400 \\
\hline 69 & R.K. & 9 & $5 \mathrm{~m}$ & A & $1(H)$ & $15( \pm)$ & 7,500 & 380 \\
\hline 70 & K.S. & कิ & $75 \mathrm{~d}$ & $\mathrm{R}$ & $5( \pm)$ & $13( \pm)$ & 6,500 & 399 \\
\hline 71 & R.M. & 우 & $7 \mathrm{~m}$ & $\mathrm{R}$ & $4( \pm)$ & $1(H)$ & 4,800 & 470 \\
\hline 72 & N.K. & 䓃 & $1 \mathrm{y} 2 \mathrm{~m}$ & $\mathrm{R}$ & $5( \pm)$ & $5( \pm)$ & 4,850 & 330 \\
\hline 73 & H.S. & 古 & $4 \mathrm{~m}$ & $\mathbf{R}$ & $15( \pm)$ & $7( \pm)$ & 4,950 & 460 \\
\hline 74 & T.I. & 9 & $6 \mathrm{~m}$ & $\mathrm{Ep}$ & $13( \pm)$ & $5( \pm)$ & 4,900 & 330 \\
\hline 75 & K. T. & s & $6 \mathrm{~m}$ & $\mathrm{~B}^{2}$ & $12( \pm)$ & $4(-)$ & 5,300 & 300 \\
\hline 76 & T.K. & $\delta$ & $1 \mathrm{y} 2 \mathrm{~m}$ & B & $14(-)$ & $2(+)$ & 5,450 & 475 \\
\hline 77 & Z. E. & 今 & $11 \mathrm{~m}$ & A & $1(+)$ & $1(+)$ & 6,850 & 270 \\
\hline 78 & T.S. & 우 & $49 \mathrm{~d}$ & Ip & $5( \pm)$ & $5( \pm)$ & 4,800 & 460 \\
\hline 79 & K.S. & $\delta$ & $7 \mathrm{~m}$ & $\mathrm{Hi}$ & $1(+1)$ & $1(H)$ & 4,910 & 330 \\
\hline 80 & M.r. & $\hat{\sigma}$ & $45 \mathrm{~d}$ & $\mathrm{Hi}$ & $16(-)$ & $6(-)$ & 4,300 & 480 \\
\hline 81 & S.I. & $\delta$ & $3 \mathrm{~m}$ & $\mathrm{R}$ & $3( \pm)$ & $16(-)$ & 5,240 & 145 \\
\hline 82 & R.K. & 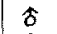 & $10 \mathrm{~m}$ & $\mathrm{Hy}_{y}$ & 5 (上) & $6(-)$ & 5,200 & 390 \\
\hline 83 & s. 0 . & 우 & $11 \mathrm{~m}$ & $\mathrm{He}$ & $1(+t)$ & $1(+)$ & 5,230 & 190 \\
\hline 84 & Y.I. & $\delta$ & 3 in & $\mathrm{B}$ & $14(-)$ & $1( \pm)$ & 4,100 & 201 \\
\hline 85 & S.S. & 今 & $41 d$ & B & $1( \pm)$ & $7(-)$ & 5,030 & 160 \\
\hline 86 & R.K. & 9 & $7 \mathrm{~m}$ & I & $9(-)$ & $9(--)$ & 4,800 & 328 \\
\hline 87 & R.K. & 우 & $7 \mathrm{~m}$ & $\mathbf{R}$ & $12(-)$ & $11( \pm)$ & 5,490 & 414 \\
\hline 88 & M.A. & s & $7 \mathrm{~m}$ & $\mathbf{P}$ & $12( \pm)$ & $1(+)$ & 4,990 & 558 \\
\hline 89 & R.O. & t & $9 \mathrm{~m}$ & I & $3(-)$ & $5( \pm)$ & 5,800 & 290 \\
\hline 90 & K.T. & $\hat{\delta}$ & $9 \mathrm{~m}$ & $I_{p}$ & $4( \pm)$ & $4( \pm)$ & $4,910^{\circ}$ & 474 \\
\hline 91 & K.0. & $\delta$ & $5 \mathrm{~m}$ & $\mathbf{E}$ & $1( \pm)$ & 1 (士) & 6,410 & 460 \\
\hline 92 & K.T. & $\delta$ & $4 \mathrm{~m}$ & $\overline{\mathrm{R}}$ & $14( \pm)$ & $14 \pm$ & 4,830 & 385 \\
\hline 93 & s. 0 . & $\delta$ & $2 \mathrm{~m}$ & Ps? & $3(-)$ & $13(-)$ & 4,100 & 365 \\
\hline 94 & T.I. & 우 & $4 \mathrm{~m}$ & $\mathbf{E}$ & $1(t)$ & $*$ & 5,210 & 390 \\
\hline 95 & S. A. & 官 & $10 \mathrm{~m}$ & Lo & $7(-)$ & $7(-)$ & 5,130 & 754 \\
\hline 96 & K.K. & 우 & $9 \mathrm{~m}$ & $\mathrm{R}$ & $7(-)$ & $16(-)$ & 5,790 & 329 \\
\hline 97 & S. H. & $\hat{s}$ & $10 \mathrm{~m}$ & $\mathrm{D}$ & $15(-)$ & $14( \pm)$ & 6,210 & 537 \\
\hline 98 & T.K. & 우 & $5 \mathrm{~m}$ & $\mathrm{Br}+\mathrm{Hi}$ & $14( \pm)$ & $14( \pm)$ & 4,680 & 364 \\
\hline 99 & S.H. & $\hat{\delta}$ & $8 \mathrm{~m}$ & D & $1(+)$ & $7(-)$ & 5,270 & 364 \\
\hline 100 & T.H. & 우 & $43 \mathrm{~d}$ & $\mathrm{Hu}$ & $8( \pm)$ & $15(-)$ & 4,050 & 388 \\
\hline 101 & Y.S. & s。 & 1 y $3 \mathrm{~m}$ & $\mathbf{P}$ & $8(-)$ & $1( \pm)$ & 4,740 & 257 \\
\hline 102 & R.S. & 우 & $46 a$ & $\mathrm{Hg}$ & $1(+)$ & $1(+)$ & 5,200 & 317 \\
\hline 103 & T.K. & 斻 & $6 \mathrm{~m}$ & $\operatorname{Im}$ & $11( \pm$ & $6(+)$ & 4,930 & 362 \\
\hline
\end{tabular}


TAble 4. Continued.

\begin{tabular}{|c|c|c|c|c|c|c|c|c|}
\hline \multirow{2}{*}{ No. } & \multirow{2}{*}{ Name } & \multirow{2}{*}{ Sex } & \multirow{2}{*}{$\begin{aligned} & \text { Age } \\
\mathrm{y} & =\text { years } \\
\mathrm{m} & =\text { months } \\
\mathrm{d} & =\text { days }\end{aligned}$} & \multirow{2}{*}{ Diagnosis§. } & \multicolumn{2}{|c|}{$\begin{array}{c}\text { Arakawa's } \\
\text { reaction }\end{array}$} & \multirow{2}{*}{$\begin{array}{c}\text { Red cell } \\
\text { count } \\
\text { per cmm. } \\
\text { (thousands) }\end{array}$} & \multirow{2}{*}{$\begin{array}{c}\text { Blood } \\
\text { platelet } \\
\text { count per } \\
\text { cmm. } \\
\text { (thousands) }\end{array}$} \\
\hline & & & & & Right & Left & & \\
\hline 104 & T. 0 . & $\hat{b}$ & $3 \mathrm{~d}$ & $\mathrm{D}$ & $1(-)$ & $10(-)$ & 4,200 & 308 \\
\hline 105 & K.M. & 令 & $4 \mathrm{~m}$ & $\mathrm{E}$ & $4(-) 1$ & $15(-m)$ & 4,740 & 392. \\
\hline 106 & T.M. & 令 & $4 \mathrm{~m}$ & $\mathrm{Br}$ & $5 \pm 1$ & $16(-)$ & 5,900 & 413 \\
\hline 107 & R.M. & ิㅇ & $3 \mathrm{~m}$ & $\mathrm{D}+\mathrm{Lc}$ & $14(-)$ & $11(-)$ & 5,300 & 180 \\
\hline 108 & S. F. & 今ิ & $4 \mathrm{~m}$ & $\mathrm{Bp}$ & $8(-)$ & $5( \pm)$ & 4,980 & 453 \\
\hline 109 & M.I. & s & $9 \mathrm{~m}$ & $\mathbf{I}+\mathbf{D}$ & $13(-)$ & $13(-)$ & 5,500 & 357 \\
\hline 110 & T.K. & $\delta$ & $2 \mathrm{~m}$ & Ht & $1( \pm) 1$ & $16 i-5$ & 3,400 & 290 \\
\hline 111 & M.S. & 우 & $11 \mathrm{~m}$ & I & $1(t)$ & $\mathbf{1}(t)$ & 5,460 & 617 \\
\hline 112 & J.S. & $\hat{\delta}$ & $7 \mathrm{~m}$ & $\mathrm{R}$ & $1( \pm)$ & $5(-)$ & 4,790 & 360 \\
\hline 113 & H.Y. & $\delta$ & $7 \mathrm{~m}$ & $\mathrm{R}$ & $16(-) 1$ & $11( \pm)$ & 4,900 & 380 \\
\hline 114 & S. 0 & 우 & $49 d$ & $\mathrm{~B}+\mathrm{St}$ & $|13(-)| 1$ & $15(-)$ & 4,400 & 290 \\
\hline 115 & M.S. & ồ & $6 \mathrm{~m}$ & $\mathbf{R}$ & $16(-)$ & $1( \pm)$ & 4,350 & 310 \\
\hline 116 & R.N. & 우 & $4 \mathrm{~m}$ & D & 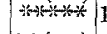 & $16(-)$ & 5,830 & 345 \\
\hline 117 & J. M. & $\delta$ & $3 \mathrm{~m}$ & Id & $16(-)$ & $1(H)$ & 4,600 & 430 \\
\hline 118 & H.T. & $\delta$ & $3 \mathrm{~m}$ & $\mathbf{R}$ & $14( \pm)$ & $14( \pm)$ & 4,830 & 385 \\
\hline 119 & T.S. & s & $11 \mathrm{~m}$ & $\mathrm{I}+\mathrm{H}$ & $7( \pm)$ & $16(-)$ & 5,270 & 250 \\
\hline 120 & K.U. & क & $9 \mathrm{~m}$ & $\mathrm{R}$ & $11(-)$ & $11(-)$ & 5,740 & 321 \\
\hline 121 & Y.M. & 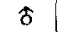 & $2 \mathrm{~m}$ & $\mathrm{H}+\mathrm{Hi}$ & $13(-)$ & $13(-)$ & 3,590 & 260 \\
\hline 122 & Y.T. & 占 & $5 \mathrm{~m}$ & $I$ & $13(-)$ & $13(-)$ & 4,750 & 314 \\
\hline 123 & K.H. & 우 & $3 \mathrm{~m}$ & D & $16(-) \mid$ & $\mid 11(--)$ & 2,450 & 198 \\
\hline 124 & K.I. & $\delta$ & $7 \mathrm{~m}$ & $\mathbf{P}$ & $15(-) \mid 1$ & $11(-)$ & 6,180 & 303 \\
\hline 125 & N.S. & 우 & $5 . \mathrm{m}$ & $D$ & $11(-)$ & $12(-)$ & 4,480 & 521 \\
\hline 126 & T.S. & 우 & $47 \mathrm{~d}$ & $\mathrm{Pe}$ & $11(-) 1$ & $16(-)$ & 4,100 & 478 \\
\hline 127 & R. Y. & ิิ & $3 \mathrm{~m}$ & $\mathrm{E}+\mathrm{Br}$ & $1(+)$ & $1(+)$ & 4,450 & 453 \\
\hline 128 & K.N. & $\delta$ & $10 \mathrm{~m}$ & $\mathrm{Bp}$ & $5(+) 1$ & $14( \pm)$ & 5,380 & 414 \\
\hline 129 & K.M. & $\delta$ & $8 \mathrm{~m}$ & $\mathrm{R}$ & $1(+) 1$ & $11(-)$ & 4,960 & 359 \\
\hline 130 & S. K. & ช & $4 \mathrm{~m}$ & $\mathrm{~Pb}$ & $9(+)$ & $7(-)$ & 4,800 & 410 \\
\hline 131 & K.I. & $\delta$ & $5 \mathrm{~m}$ & A & $3(-)$ & $\mathbf{1}( \pm)$ & 4,710 & 350 \\
\hline 132 & N.K. & $\hat{\delta}$ & $9 \mathrm{~m}$ & $\mathrm{R}$ & $3(+)$ & $5(+)$ & 5,800 & 440 \\
\hline 133 & K.O. & 本 & $4 \mathrm{~m}$ & $\mathrm{~B}$ & $14(-)$ & $7(-)$ & 4,350 & 660 \\
\hline 134 & M.M. & 우 & $3 \mathrm{~m}$ & Ib & $11(+) 1$ & $11(t)$ & 5,000 & 950 \\
\hline 135 & K.T. & के & $2 \mathrm{~m}$ & A & $11(-)$ & $16(-)$ & 5,800 & 481 \\
\hline 136 & K.K. & . & $10 \mathrm{~m}$ & $\mathrm{Pc}$ & $6( \pm)$ & $6( \pm)$ & 4,510 & 550 \\
\hline 137 & J. W. & $\delta$ & I y $4 \mathrm{~m}$ & Ep & $3(+)$ & $3(+)$ & 4,450 & 790 \\
\hline 138 & K.T. & s & $5 \mathrm{~m}$ & $\mathrm{P}$ & $2( \pm\}$ & $2( \pm)$ & 6,200 & 382 \\
\hline 139 & Y.I. & $\hat{o}$ & $3 \mathrm{~m}$ & B & $1(+t)$ & $1(+)$ & 4,100 & 200 \\
\hline 140 & R.G. & $\hat{\sigma}$ & $5 \mathrm{~m}$ & $\mathbf{R}$ & $1( \pm)$ & $1( \pm)$ & 4,930 & 370 \\
\hline & $\S$ & Foc & ot note to Tab & 2. & & & . & \\
\hline & & $2^{\prime}$ & $3^{\prime} \quad 4^{\prime} \quad 5^{\prime}$ & & & & & \\
\hline & $*$ & $+\mathbb{H}$ & H H H H & & & & & \\
\hline & ** & \pm+ & Hit H & & & & & \\
\hline & $* * *$ & - + & Ht H H & & & & & \\
\hline & 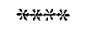 & $H+$ & $H++$ & & & & & \\
\hline & 1 & \pm+ & $H$ H H & & & & & \\
\hline
\end{tabular}

difference of thrombocyte count was much more striking than that of red cell count.

3. Distribution of Arakawa-positive, intermediate and negative groups according to blood platelet count (Cf. Tables 10 and 11). 
Table 5 .

Cases with intermediate Arakawa's reaction in which infants and/or their mothers had been taking vitamin $B$.

\begin{tabular}{|c|c|c|c|c|c|c|c|c|c|c|}
\hline \multirow{2}{*}{$\dot{0}$} & \multirow[t]{2}{*}{ Name } & \multirow{2}{*}{ 离 } & \multirow{2}{*}{$\begin{array}{c}\text { Age } \\
\mathrm{y}=\text { years } \\
\mathrm{m}=\text { months } \\
\mathrm{d}=\text { days }\end{array}$} & \multirow{2}{*}{ 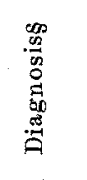 } & \multicolumn{2}{|c|}{$\begin{array}{c}\text { Arakawa's } \\
\text { reaction }\end{array}$} & \multirow{2}{*}{$\begin{array}{l}\text { Red cell } \\
\text { count } \\
\text { per cmm. } \\
\text { (thou- } \\
\text { sands) }\end{array}$} & \multirow{2}{*}{$\begin{array}{l}\text { Blood } \\
\text { platelet } \\
\text { count } \\
\text { per cmm. } \\
\text { (thou- } \\
\text { sands) }\end{array}$} & \multicolumn{2}{|c|}{$\begin{array}{l}\text { Administra- } \\
\text { tion of } \\
\text { vitamin } B \text { to }\end{array}$} \\
\hline & & & & & Right & Left & & & Mother & Infa \\
\hline 1 & T. $\mathrm{Y}$ & $\delta$ & $6 \mathrm{~m}$ & $\mathrm{Im}$ & $3( \pm)$ & $3(+)$ & 5,140 & 415 & - & + \\
\hline 2 & R.S. & $\delta$ & $12 \mathrm{~m}$ & B & $15( \pm)$ & $13 \pm 1 \pm$ & 5,800 & 480 & - & + \\
\hline 3 & S. 0 & 今 & $2 \mathrm{~m}$ & Ps? & $7( \pm)$ & $4(-)$ & 4,200 & 351 & + & + \\
\hline 4 & T.K. & 8 & $6 \mathrm{~m}$ & $\operatorname{Im}$ & $7( \pm)$ & $6(+)$ & 4,930 & 362 & $\therefore$ & + \\
\hline 5 & T.A. & $\diamond$ & $3 \mathrm{~m}$ & An & $11(-)$ & $11(-)$ & 4,630 & 219 & + & + \\
\hline 6 & S. F. & $\hat{\delta}$ & $4 \mathrm{~m}$ & $\mathrm{Bp}$ & $3( \pm)$ & $2(-)$ & 4,650 & 530 & + & + \\
\hline 7 & T.A. & 오 & $8 \mathrm{~m}$ & $\mathrm{D}$ & $4(-)$ & $4(-)$ & 5,150 & 267 & + & + \\
\hline 8 & M.I. & 今) & $9 \mathrm{~m}$ & $D$ & it & * & 5,450 & 279 & + & + \\
\hline 9 & $\mathbf{Y} . \mathbf{Y}$. & 우 & $34 d$ & $\mathrm{Br}$ & $6(-)$ & $14(-)$ & 4,920 & 320 & + & + \\
\hline 10 & K.Y. & $\%$ & $7 \mathrm{~m}$ & $\mathbf{R}$ & $14(-)$ & $6(+)$ & 5,640 & 226 & + & + \\
\hline 11 & M.K. & s. & $5 \mathrm{~m}$ & A & $10( \pm)$ & $7(-)$ & 5,530 & 403 & + & + \\
\hline 12 & K.N. & $\hat{0}$ & $1 \mathrm{~g} 1 \mathrm{~m}$ & $\mathrm{Br}$ & $1(+)$ & $7( \pm)$ & 4,500 & 343 & + & + \\
\hline 13 & E.I. & $\Delta$ & $9 \mathrm{~m}$ & I & $*$ & $11( \pm)$ & 5,240 & 293 & + & + \\
\hline 14 & S. H. & $\delta$ & $4 \mathrm{~m}$ & $\mathrm{R}$ & $3( \pm)$ & $4(-)$ & 4,770 & 363 & + & + \\
\hline 15 & H.T. & $\delta$ & $5 \mathrm{~m}$ & $\mathbf{D}$ & $11(-)$ & $6( \pm 5)$ & 4,220 & 283 & - & + \\
\hline 16 & F.M. & $\delta$ & $1 \mathrm{~s} 3 \mathrm{~m}$ & $\mathrm{Bp}$ & $7(-)$ & $7(-)$ & 5,060 & 448 & + & + \\
\hline 17. & H.U. & $\delta$ & $10 \mathrm{~m}$ & $\mathrm{Br}+\mathrm{D}$ & $9(-j$ & $9(-)$ & 4,300 & 349 & + & + \\
\hline 18 & K.I. & 苔 & 7 in & $\mathrm{P}$ & $|10(-)|$ & $3( \pm)$ & 4,930 & 345 & $\cdots$ & + \\
\hline 19 & M.M. & 우 & $4 \mathrm{~m}$ & $\mathrm{D}$ & 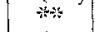 & $2(--)$ & 4,750 & 415 & + & + \\
\hline 20 & A.N. & 우 & $1 \mathrm{y} 3 \mathrm{~m}$ & $\mathbf{R}$ & $8( \pm)$ & $10(-m)$ & 5,190 & 326 & - & + \\
\hline 21 & M.I. & $\delta$ & $9 \mathrm{~m}$ & D & $14(-)$ & $1(+)$ & 4,980 & 290 & + & + \\
\hline 22 & S. U. & $\widehat{\delta}$ & $10 \mathrm{~m}$ & $\mathrm{Br}$ & $8(+)$ & $3(-)$ & 5,050 & 362 & + & -1 \\
\hline 23 & N.S. & 우 & $53 \mathrm{~d}$ & $P$ & $|11(+)|$ & $11(+)$ & 5,200 & 580 & + & + \\
\hline 24 & S.M. & 定 & $3 \mathrm{~m}$ & $\mathrm{Hu}$ & $5( \pm)$ & $15( \pm)$ & 5,800 & 560 & + & + \\
\hline 25 & J. 0. & $\delta$ & $2 \mathrm{~m}$ & Le & $16(-)$ & $14( \pm)$ & 6,500 & 271 & + & + \\
\hline 26 & T.K. & 0 & $9 \mathrm{~m}$ & $\mathbf{R}$ & $1(+)$ & $1(+)$ & 6,950 & 350 & - & + \\
\hline 27 & K.S. & 우 & $6 \mathrm{~m}$ & 1 & $7( \pm)$ & $7( \pm)$ & 5,250 & 340 & - & + \\
\hline 28 & Y.I. & 우 & $6 \mathrm{~m}$ & $\mathrm{Bp}$ & $15(-)$ & $7(-)$ & 8,100 & 370 & - & + \\
\hline 29 & K.T. & 우 & $8 \mathrm{~m}$ & D & $6( \pm)$ & $4(-)$ & 5,850 & 360 & + & + \\
\hline 30 & K.S. & 우 & $1 \mathrm{~g} 2 \mathrm{~m}$ & $N$ & $7(+)$ & $7(+)$ & 4,350 & 351 & - & + \\
\hline 31 & K.T. & $\delta$ & $5 \mathrm{~m}$ & B & $16(-)$ & $6(-)$ & 4,800 & 420 & + & + \\
\hline 32 & A.I. & 8 & $9 \mathrm{~m}$ & $\mathrm{D}$ & $13( \pm)$ & $2(+)$ & 6,600 & 400 & + & + \\
\hline 33 & K.I. & 우 & $1 \mathrm{y} 1 \mathrm{~m}$ & $\mathrm{Ep}_{p}$ & $2(+)$ & $2(+)$ & 5,750 & 300 & + & f \\
\hline 34 & S.I. & $\hat{0}$ & $1 \mathrm{y} 4 \mathrm{~m}$ & D & $1(+)$ & $1(+)$ & 6,350 & 201 & - & + \\
\hline 35 & K.S. & 本 & $1 \mathrm{y} 4 \mathrm{~m}$ & $\mathrm{D}+\mathrm{Dc}$ & $14(-)$ & $2(-)$ & 5,700 & 450 & $-\cdots$ & + \\
\hline 36 & K.G. & 1 & $12 \mathrm{~m}$ & $B$ & $16(-)$ & $12( \pm)$ & 5,140 & 502 & + & + \\
\hline 37 & J.T. & 官 & $4 \mathrm{~m}$ & B & $16(-)$ & $14( \pm)$ & 4,450 & 451 & + & + \\
\hline 38 & N.S. & 우 & $61 d$ & $\mathrm{P}$ & $2(+)$ & $5(H)$ & 4,300 & 450 & + & + \\
\hline 39 & K.T. & 우 & $9 \mathrm{~m}$ & D. & $8(-)$ & $8(-)$ & 7,100 & 340 & + & + \\
\hline 40 & K.S. & 今 & $8 \mathrm{~m}$ & Ps? & 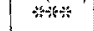 & $1( \pm)$ & 5,250 & 220 & + & + \\
\hline 41 & S. S. & $\delta$ & $3 \mathrm{~m}$ & $\mathrm{E}$ & $2(-)$ & $|11( \pm)|$ & 4,670 & 280 & + & + \\
\hline 42 & $\mathbf{Y} . \mathbf{T}$. & 우 & $11 \mathrm{~m}$ & $B p$ & $3( \pm)$ & $1(+)$ & 4,270 & 490 & - & + \\
\hline 43 & M.F. & q & $9 \mathrm{~m}$ & $\mathrm{H}$ & $3( \pm)$ & $1(+)$ & 4,270 & 491 & - & + \\
\hline 44 & S. K. & 实 & $2 \mathrm{~m}$ & Ip & $7(-)$ & $6( \pm)$ & 3,930 & 264 & - & + \\
\hline 45 & S. Y. & $\hat{\delta}$ & $6 \mathrm{~m}$ & D & $1( \pm)$ & $|14(-)|$ & 5,040 & 514 & - & + \\
\hline 46 & N.W. & 9 & $1 \mathrm{y} 1 \mathrm{~m}$ & $D$ & $1( \pm)$ & $14(-)$ & 6,100 & 301 & - & + \\
\hline 47 & K.T. & $f$ & $10 \mathrm{~m}$ & $\mathrm{Hm}$ & $1(H)$ & $3( \pm)$ & 5,220 & 277 & - & + \\
\hline 48 & J. I. & $\delta$ & $3 \mathrm{~m}$ & $\mathrm{H}$ & $16(-)$ & $11( \pm)$ & 3,530 & 193 & - & + \\
\hline 49 & H.K. & 우 & $1 \mathrm{y}$ & $\mathrm{Bp}$ & $1( \pm)$ & $|x(H)|$ & 5,520 & 290 & - & $t$ \\
\hline
\end{tabular}


Table 5. Continued.

\begin{tabular}{|c|c|c|c|c|c|c|c|c|c|c|}
\hline \multirow{2}{*}{$\dot{0}$} & \multirow{2}{*}{ Name } & \multirow{2}{*}{$\mathbb{D}_{0}^{x}$} & \multirow{2}{*}{$\begin{array}{l}\quad \text { Age } \\
\mathrm{y}=\text { years } \\
\mathrm{m}=\text { months } \\
\mathrm{d}=\text { days }\end{array}$} & \multirow{2}{*}{ 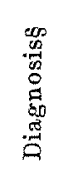 } & \multicolumn{2}{|c|}{$\begin{array}{l}\text { Arakawa's } \\
\text { reaction }\end{array}$} & \multirow{2}{*}{$\begin{array}{c}\text { Red cell } \\
\text { count } \\
\text { per cmm. } \\
\text { (thou- } \\
\text { sands) }\end{array}$} & \multirow{2}{*}{$\begin{array}{l}\text { Blood } \\
\text { plate } \\
\text { count } \\
\text { per emm. } \\
\text { (thou- } \\
\text { sands) }\end{array}$} & \multicolumn{2}{|c|}{$\begin{array}{l}\text { Administra- } \\
\text { tion of } \\
\text { vitamin B to }\end{array}$} \\
\hline & & & & & Right & Left & & & Mother & Infant \\
\hline 50 & S.N. & 今ิ & 9 & D & $1( \pm)$ & $1( \pm)$ & 4,490 & 280 & - & + \\
\hline 51 & M.M. & 8 & $6 \mathrm{~m}$ & $D$ & $12(-)$ & $11( \pm)$ & 5,350 & 221 & - & + \\
\hline 52 & Y.K. & 우 & $3 \mathrm{~m}$ & Wn & $10( \pm)$ & $15( \pm)$ & 4,260 & 290 & + & + \\
\hline 53 & N.T. & 우 & $1 \mathrm{y} 1 \mathrm{~m}$ & $\mathrm{D}$ & 年 & $1( \pm)$ & 5,680 & 251 & - & + \\
\hline 54 & H.Y. & 㕝 & $7 \mathrm{~m}$ & $\mathrm{R}$ & $|14(-)|$ & $6(-)$ & 5,640 & 226 & + & + \\
\hline
\end{tabular}

$\$$ Cf. Foot note to Table 2.

$$
\begin{aligned}
& \begin{array}{lllll}
1^{\prime} & 2^{\prime} & 3^{\prime} & 4^{\prime} & 5^{\prime}
\end{array} \\
& * \pm+H+H \\
& * 2+4+4+
\end{aligned}
$$

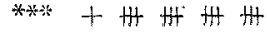

$$
\begin{aligned}
& \text { 然然士 }+ \text { H H H }
\end{aligned}
$$

One will see by glancing over Tables 10 and 11, that most cases of the strongly or normally Arakawa-positive group fall in the least number (0-350 thousands) of thrombocytes; as to the subgroup with vitamin $B$, all the cases fall in the least number. There is not a single case belonging to the submaximal (551-650 thousands) or to the maximal (over 650 thousands) of thrombocytes in the strongly or normally Arakawa-positive group, whereas in the completely or almost completely Arakawa-negative group, $10 \%$ of the subgroup without vitamin $B$ belong to the maximal number of thrombocytes. But in each group the subgroup with vitamin B shows an inclination to move to the small number of thrombocytes.

\section{Comment.}

The normal platelet count of an infants, according to Baar and Stransky ${ }^{13)}$ is 200-300 thousands, and according to Keilmann ${ }^{14)}$ the count is a very low one $48-192$ thousands. Jarch ${ }^{15)}$ reported that "blood platelet count in normal newborn infants may show wide variation. In the majority of cases, the count is between 150,000 and 250,000." Merrit and Davids on ${ }^{16)}$ stated that blood platelet at

13) H. Baar and E. Stransky, Die klinische Hämatologie des Kinderalters, Leipzig and Wien 1928, 44.

14) K. Keilma n $n$, Mschr. f. Kinderheilk., 1922, 23, 383.

15) J. J a rch o, Arch. Pediatr., 1930, 47, 230.

16) K. Merrit and T. D avid so n, Am. J. Dis. Child., 1933, 46, 990. 
TABLE 6.

Arakawa-negative cases in which infants and their mothers had not been taking vitamin B preparation.

\begin{tabular}{|c|c|c|c|c|c|c|c|c|}
\hline \multirow{2}{*}{ No. } & \multirow{2}{*}{ Name } & \multirow{2}{*}{$\operatorname{Sex}$} & \multirow{2}{*}{$\begin{aligned} & \text { Age } \\
y & =\text { years } \\
\mathrm{m} & =\text { months } \\
\mathrm{d} & =\text { days }\end{aligned}$} & \multirow{2}{*}{ Diagnosis* } & \multicolumn{2}{|c|}{$\begin{array}{c}\text { Arakawa's } \\
\text { reaction }\end{array}$} & \multirow{2}{*}{$\begin{array}{c}\text { Rell cell } \\
\text { count } \\
\text { per cmm. } \\
\text { (thousands) }\end{array}$} & \multirow{2}{*}{$\begin{array}{l}\text { Blood plate- } \\
\text { let count } \\
\text { per cmm. } \\
\text { (thousands) }\end{array}$} \\
\hline & & & & & Right & Left & & \\
\hline 1 & N.T. & $\hat{\delta}$ & $1 \mathrm{y} 1 \mathrm{~m}$ & $D$ & $|14(-)|$ & $16(-)$ & 4,310 & 520 \\
\hline 2 & М.H. & * & $1 \mathrm{y} 3 \mathrm{~m}$ & $\mathrm{D}$ & $14(-)$ & $14(-)$ & 5,210 & 441 \\
\hline 3 & K. 0 & $q$ & $50 \mathrm{~d}$ & $\mathbf{H}$ & $8(-)$ & $16(-)$ & 4,430 & 410 \\
\hline 4 & Y.S. & s & $65 d$ & $\mathrm{~A}+\mathrm{He}$ & $16(-)$ & $16(-)$ & 4,220 & 540 \\
\hline 5 & K.H. & 今 & $1 \mathrm{y} 1 \mathrm{~m}$ & D & $16(-3$ & $15(-)$ & 6,400 & 480 \\
\hline 6 & I. H. & $\delta$ & $49 \mathrm{~d}$ & Ib & $16(-) \mid$ & $16(-)$ & 4,600 & 513 \\
\hline 7 & J. K. & 今 & $9 \mathrm{~m}$ & $\mathrm{D}$ & $16(-)$ & $15(-)$ & 8,560 & 480 \\
\hline 8 & M.K. & 운 & $1 \mathrm{y} 5 \mathrm{~m}$ & B & $16(-)$ & $15( \pm)$ & 5,200 & 599 \\
\hline 9 & K.H. & 우 & $1 \mathrm{y} 2 \mathrm{~m}$ & $\mathbf{R}$ & $16(-)$ & $8(-)$ & 4,900 & 468 \\
\hline 10 & Y.o. & 우 & $3 \mathrm{~m}$ & $\mathrm{P}$ ? & $16(-)$ & $15(-)$ & 5,650 & 580 \\
\hline 11 & S.S. & 오 & $40 \mathrm{~d}$ & $\mathbf{I} p$ & $14(-3)$ & $14(-3)$ & 4,800 & 271 \\
\hline 12 & M.M. & ơ & $27 d$ & Ip & $16(-)$ & $16(-)$ & 5,050 & 560 \\
\hline 13 & Y.Y. & $\hat{\delta}$ & $1 \mathrm{y} 6 \mathrm{~m}$ & $\mathrm{R}$ & $16(-5)$ & $16(-)$ & 5,300 & 562 \\
\hline 14 & M.S. & 8 & $43 \mathrm{~d}$ & Ip & $16(-)$ & $16(-)$ & 4,200 & 350 \\
\hline 15 & K.T. & $\delta$ & $5 \mathrm{mo}$ & B & $16(-)$ & $15(-)$ & 5,000 & 520 \\
\hline 16 & Y.O. & tิ & $1 \mathrm{y} 3 \mathrm{~m}$ & $\mathbf{R}$ & $16(-m)$ & $16(-)$ & 5,900 & 249 \\
\hline 17 & K.O. & $\delta$ & $5 \mathrm{~m}$ & Ip & $15(-5)$ & $15(-)$ & 5,850 & 1.080 \\
\hline 18 & S. T. & $\delta$ & $10 \mathrm{~m}$ & B & $16(-)$ & $16(-)$ & 4,850 & 379 \\
\hline 19 & S. W. & 우 & $6 \mathrm{~m}$ & $\mathrm{D}$ & $15(-)$ & $|15(-\cdots)|$ & 4,850 & 485 \\
\hline 20 & A.K. & 우 & $8 \mathrm{~m}$ & A & $8(-)$ & $8(-)$ & 6,960 & 490 \\
\hline 21 & S. A. & $\delta$ & $3 \mathrm{~m}$ & H & $16(-)$ & $|16(-)|$ & 3,700 & 418 \\
\hline 22 & M.S. & 우 & $2 \mathrm{~m}$ & Ip & $14(-)$ & $16(-)$ & 3,900 & 341 \\
\hline 23 & S. A. & 2 & $9 \mathrm{~m}$ & An & $15( \pm)$ & $16(-)$ & 4,550 & 220 \\
\hline 24 & R.S. & $\delta$ & $1 \mathrm{y}$ & $B$ & $16(-)$ & $16(-)$ & 4,050 & 606 \\
\hline 25 & K.H. & 우 & $3 \mathrm{~m}$ & $\mathbf{R}$ & $16(-)$ & $14(-)$ & 3,150 & 350 \\
\hline 26 & S.H. & $\delta$ & $11 \mathrm{~m}$ & $\mathrm{Hm}$ & $16(-)$ & $16(-)$ & 5,690 & 410 \\
\hline 27 & К.O. & $\hat{\delta}$ & $4 \mathrm{~m}$ & Ip & $16(-)$ & $16(-)$ & 4,580 & 430 \\
\hline 28 & $\mathrm{~J} . \mathrm{K}$. & 舍 & $5 \mathrm{~m}$ & $\mathrm{Br}$ & $8(-)$ & $15( \pm\}$ & 5,500 & 327 \\
\hline 29 & K.S. & $\delta$ & $9 \mathrm{~m}$ & I & $16(-) \mid$ & $16(-)$ & 4,730 & 329 \\
\hline 30 & Y.N. & 9 & $4 \mathrm{~m}$ & $\bar{D}$ & $16(-)$ & $16(-)$ & 4,480 & 454. \\
\hline 31 & K.Y. & $q$ & $3 \mathrm{~m}$ & A & $16(-)$ & $16(-)$ & 4,460 & 429 \\
\hline 32 & T. A. & $\hat{\delta}$ & $2 \mathrm{~m}$ & An & $16(-)$ & $16(-)$ & 4,780 & 300 \\
\hline 33 & T.A. & 우 & $8 \mathrm{~m}$ & $I-D$ & $16(-)$ & $16(-)$ & 5,370 & 507 \\
\hline 34 & M.S. & 우 & $5 \mathrm{~m}$ & $\mathrm{Br}$ & $16(-)$ & $8(-)$ & 4,880 & 479 \\
\hline 35 & S. M. & 우 & $1 \mathrm{y} 1 \mathrm{~m}$ & $\mathrm{D}$ & $16(-)$ & $15(-)$ & 4,930 & 301 \\
\hline 36 & K.o. & 古 & $2 \mathrm{~m}$ & $\mathrm{Hu}$ & $14(-)$ & $16(-)$ & 4,900 & 344 \\
\hline 37 & $\mathbf{Y . Y .}$ & 우 & $25 d$ & $\mathrm{Br}$ & $16(-)$ & $16(-)$ & 5,100 & 349 \\
\hline 38 & S.M. & $\delta$ & $5 \mathrm{~m}$ & $\mathrm{Br}$ & $16(-)$ & $16(-)$ & 5,450 & 611 \\
\hline 39 & T.T. & $\delta$ & $4 \mathrm{~m}$ & $\mathbf{R}$ & $15(-)$ & $15(-5)$ & 5,290 & 397 \\
\hline 40 & S. K. & s & $50 \mathrm{~d}$ & $\mathrm{Hi}+\mathrm{Hu}$ & $16(-)$ & $16(-)$ & 3,650 & 232 \\
\hline 41 & 'M.I. & 우 & $54 \mathrm{~d}$ & $\mathrm{Hu}$ & $16(-)$ & $|15(-)|$ & 4,300 & 323 \\
\hline 42 & S. T. & $\delta$ & $4 \mathrm{~m}$ & $\mathrm{D}$ & $15(-)$ & $15(-) \mid$ & 4,750 & 273 \\
\hline 43 & J. T. & 8 & $31 \mathrm{~d}$ & $\mathbf{H}$ & $16(-)$ & $16(-)$ & 5,700 & 390 \\
\hline 44 & T.M. & 우 & $2 \mathrm{~m}$ & $\mathbf{P}$ & $16(-)$ & $|16(-)|$ & 6,860 & 536 \\
\hline 45 & T.W. & 우 & $3 \mathrm{~m}$ & Bp & $16 i-5$ & $|16(-)|$ & 4,490 & 818 \\
\hline 46 & K.N. & is & $1 \mathrm{y} 1 \mathrm{~m}$ & $\mathrm{Br}$ & $12(-)$ & $6(-)$ & 5,250 & 709 \\
\hline 47 & T.K. & $\hat{\delta}$ & $9 \mathrm{~m}$ & $\mathbf{R}$ & $12(-)$ & $|16(-)|$ & 5,910 & 445 \\
\hline 48 & K.H. & 우 & $6 \mathrm{~m}$ & An & $16(-)$ & $16(-)$ & 7,600 & 508 \\
\hline 49 & K.M. & s & $1 \mathrm{y}$ & $\mathrm{H}+\mathrm{Hi}$ & $16(-)$ & $16(-)$ & 4,250 & 270 \\
\hline 50 & K.N. & 实 & $10 \mathrm{~m}$ & $\mathrm{Cn}$ & $16(-)$ & $|16(-)|$ & 4,830 & 299 \\
\hline
\end{tabular}


Table 6. Continued.

\begin{tabular}{|c|c|c|c|c|c|c|c|c|}
\hline \multirow{2}{*}{ No. } & \multirow{2}{*}{ Name } & \multirow{2}{*}{ Sex } & \multirow{2}{*}{$\begin{array}{c}\text { Age } \\
y=\text { years } \\
\mathrm{m}=\text { months } \\
\mathrm{d}=\text { days }\end{array}$} & \multirow{2}{*}{ Diagnosis* } & \multicolumn{2}{|c|}{$\begin{array}{l}\text { A rakawa's } \\
\text { reaction }\end{array}$} & \multirow{2}{*}{$\begin{array}{c}\text { Red cell } \\
\text { connt } \\
\text { per cmrn. } \\
\text { (thousands) }\end{array}$} & \multirow{2}{*}{$\begin{array}{l}\text { Blood plate } \\
\text { let comnt } \\
\text { per cmm. } \\
\text { (thousánds) }\end{array}$} \\
\hline & & & & & Right & Left & & \\
\hline 51 & S. 0 . & $\delta$ & $1 \mathrm{y}$ & $\mathrm{Bp}$ & $16(-)$ & $15(-)$ & 4,980 & 401 \\
\hline 52 & M.M. & \& & $4 \mathrm{~m}$ & $\mathrm{D}$ & $16(-)$ & $16(-)$ & 4,650 & 770 \\
\hline 53 & X.U. & 우 & $51 d$ & B & $16(-)$ & $16(-)$ & 5,170 & 582 \\
\hline 54 & S. T. & $\delta$ & $52 d$ & $\mathrm{Br}$ & $16(-)$ & $16(-)$ & 4,380 & 280 \\
\hline 55 & N.0. & 오 & $4 \mathrm{~m}$ & $\mathbf{P b}$ & $15(-)$ & $16(-)$ & 4,670 & 421 \\
\hline 56 & H.I. & i & $10 \mathrm{~m}$ & Bp & $16(-)$ & $16(-)$ & 4,800 & 503 \\
\hline 57 & I. $H$. & f & $49 \mathrm{~d}$ & $\mathrm{Ib}$ & $16(-)$ & $16(-)$ & 4,600 & 510 \\
\hline 58 & N.S. & 우 & $41 \mathrm{~d}$ & $\mathbf{P}$ & $16(-)$ & $16(-)$ & 5,300 & 700 \\
\hline 59 & H.H. & t & $2 \mathrm{~m}$ & I $p$ & $16(--m)$ & $16(-)$ & 4,500 & 751 \\
\hline 60 & S. M. & $\delta$ & $3 \mathrm{~m}$ & $\mathrm{Ip}+\mathrm{Hu}$ & $14(-)$ & $16(-)$ & 5,300 & 830 \\
\hline 61 & K.T. & $\$$ & $4 \mathrm{~m}$ & $\mathrm{Ba}$ & $16(-)$ & $16(-)$ & 4,450 & 499 \\
\hline 62 & K.T. & $\delta$ & $5 \mathrm{~m}$ & B & $16(-)$ & $15(-)$ & 5,000 & 520 \\
\hline 63 & H.O. & 乱 & $1 \mathrm{y}$ & $\mathrm{R}+\mathrm{Hi}$ & $\mid 15(-)$ & $|15(-)|$ & 5,800 & 344 \\
\hline 64 & A.I. & $\delta$ & $9 \mathrm{~m}$ & $\mathbf{D}$ & $15(-)$ & $16(-)$ & 4,300 & 320 \\
\hline 65 & J.T. & $\hat{\delta}$ & $10 \mathrm{~m}$ & $\mathrm{D}$ & $16(--)$ & $15(-)$ & 5,550 & 380 \\
\hline 66 & H.T. & 우 & $3 \mathrm{~m}$ & $\mathbf{B}$ & $15( \pm)$ & $16(-)$ & 6,300 & 600 \\
\hline 67 & S. W. & 우 & $3 \mathrm{~m}$ & D & $16(-)$ & $15(-)$ & 4,600 & 501 \\
\hline 68 & M.M. & 우 & $6 \mathrm{~m}$ & Ib & $15(-)$ & $8(-)$ & 5,410 & 570 \\
\hline 69 & G.H. & 象 & 15 & $\mathrm{R}+\mathrm{Lc}$ & $16(-)$ & $16(-)$ & 5,250 & 351 \\
\hline 70 & Y.I. & $\hat{0}$ & $3 \mathrm{in}$ & $\mathrm{H}$ & $16(-)$ & $16(-)$ & 3,240 & 663 \\
\hline 71 & T.s. & 우 & $4 \mathrm{~m}$ & $\mathrm{H}$ & $15(-)$ & $16(-)$ & 3,820 & 502 \\
\hline 72 & S. $\mathbb{E}$. & $\hat{0}$ & $7 \mathrm{~m}$ & Ip & $14-5$ & $16(-)$ & 4,580 & 351 \\
\hline 73 & R.G. & $\delta$ & $2 \mathrm{~m}$ & $\mathrm{Le}$ & $16(-)$ & $16(-)$ & 4,690 & 463 \\
\hline 74 & N.W. & 우 & $1 \mathrm{y} 1 \mathrm{~m}$ & $\mathrm{D}+\mathrm{DC}$ & $16(-)$ & $16(-)$ & 5,780 & 422 \\
\hline 75 & S. 0 & $-\delta$ & $10 \mathrm{~m}$ & $\mathbf{H} \mathbf{u}+\mathbf{A}$ & $16(-)$ & $16(-)$ & 4,380 & 398 \\
\hline 76 & K.M. & 우 & $38 d$ & $\mathrm{~B}$ & $16(-)$ & $16(-)$ & 5,490 & 340 \\
\hline 77 & K.S. & t & $5 \mathrm{~m}$ & B & $16(-)$ & $15(-)$ & 5,250 & 391 \\
\hline 78 & A.S. & 우 & $59 d$ & $\mathrm{Hmn}$ & $14(-)$ & $14(-)$ & 4,270 & 503 \\
\hline 79 & S.S. & 우 & $3 \mathrm{~m}$ & $\mathrm{Bp}$ & $16(-)$ & $15(-)$ & 2,450 & 490 \\
\hline 80 & S.N. & 今 & $46 \mathrm{~d}$ & $\mathrm{Pe}+\mathrm{Le}$ & $15(-)$ & $15(-)^{3}$ & 3,050 & 256 \\
\hline 81 & T. T. & 우 & $1 \mathrm{y}$ & $D$ & $12(-) \mid$ & $12(-)$ & 4,500 & 463 \\
\hline 82 & A.H. & 占 & $2 \mathrm{~m}$ & Wn & $16(-)$ & $8(-)$ & 3,600 & 300 \\
\hline 83 & Y.K. & \$ & $2 \mathrm{~m}$ & D & $15(-)$ & $15(-)$ & 3,900 & 209 \\
\hline 84 & Y.T. & $\delta$ & $47 \mathrm{~d}$ & $\mathbf{R}$ & $15(-)$ & $16(-)$ & 4,340 & 311 \\
\hline 85 & T.H. & 우 & $8 \mathrm{~m}$ & $\mathrm{D}$ & $14(-)$ & $15(-)$ & 5,420 & 510 \\
\hline 86 & T.M. & क & $10 \mathrm{~m}$ & $\mathrm{D}$ & $12(-)$ & $15(-)$ & 5,250 & 390 \\
\hline 87 & Y.N. & 우 & $2 \mathrm{~m}$ & $\mathrm{H}+\mathrm{E}$ & $\mid 16(--)$ & $15( \pm)$ & 3,580 & 299 \\
\hline 88 & M.M. & 오 & $27 \mathrm{~d}$ & $\mathrm{Ip}$ & $16(--)$ & $16(-)$ & 5,050 & 560 \\
\hline 89 & Y.T. & $\delta$ & $1 \mathrm{y}$ & $D$ & $12(-)$ & $12(-)$ & 5,820 & 473 \\
\hline 90 & R.K. & 오 & $1 \mathrm{y} 2 \mathrm{~m}$ & $R$ & $16(-)$ & $16(-)$ & 4,580 & 423 \\
\hline 91 & T. 0. & 우 & $6 \mathrm{~m}$ & $\mathrm{Br}$ & $|12(-)|$ & $12(-) \mid$ & 6,700 & 760 \\
\hline
\end{tabular}

birth were found to have a mean value of 227,000 . After a rise in the third month, their mean value throughout the remainder of the first year is 344,000 with a standard variation of 66,000 . In the cases of Japanese infants, Sakai's ${ }^{17)}$ count is a very high one, namely $700.5-$

17) M. Sakai, Zika Zassi, 1920, No. 245, 831 . 
TABLE 7.

Arakawa-negative cases in which infants and/or their mothers had been taking vitamin $B$ preparation.

\begin{tabular}{|c|c|c|c|c|c|c|c|c|c|c|}
\hline \multirow{2}{*}{ 完 } & \multirow{2}{*}{ Name } & \multirow{2}{*}{ 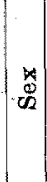 } & \multirow{2}{*}{$\begin{array}{c}\text { Age } \\
y=\text { years } \\
\mathrm{m}=\text { months } \\
d=\text { days }\end{array}$} & \multirow{2}{*}{ 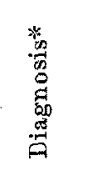 } & \multicolumn{2}{|c|}{$\begin{array}{c}\text { Arakawa's } \\
\text { reaction }\end{array}$} & \multirow{2}{*}{$\begin{array}{c}\text { Red cell } \\
\text { count } \\
\text { per cmm. } \\
\text { (thou- } \\
\text { sands) }\end{array}$} & \multirow{2}{*}{$\begin{array}{l}\text { Blood } \\
\text { platelet } \\
\text { count } \\
\text { per cmm. } \\
\text { (thou- } \\
\text { sands) }\end{array}$} & \multicolumn{2}{|c|}{$\begin{array}{l}\text { Administra- } \\
\text { tion of } \\
\text { vitamin } B\end{array}$} \\
\hline & & & & & Right & Left & & & Mother & Infant \\
\hline 1 & K.U. & $\delta$ & $37 d$ & $\mathbf{H}$ & $12(-$ & $12(-)$ & 3,250 & 260 & + & - \\
\hline 2 & M.M. & 우 & $3 \mathrm{~m}$ & $I p$ & $14(-$ & $14(-)$ & 5,500 & 330 & + & - \\
\hline 3 & K.T. & 우 & $4 \mathrm{~m}$ & D & $16(-)$ & $16(-)$ & 4,900 & 440 & - & + \\
\hline 4 & H.K. & 各 & $35 d$ & A & $16(-)$ & $16(-)$ & 5,600 & 410 & + & - \\
\hline 5 & S. A. & 今 & $3 \mathrm{~m}$ & $\mathbf{H}$ & $16(-)$ & $14(-)$ & 3,950 & 390 & - & + \\
\hline 6 & Y.H. & 古 & $9 \mathrm{~m}$ & $\mathrm{H}$ & $16(-)$ & $16(-)$ & 6,100 & 440 & + & + \\
\hline 7 & T.M. & 令 & $4 \mathrm{~m}$ & $B r$ & $16(-)$ & $16(-)$ & $\$, 800$ & 443 & + & + \\
\hline 8 & R.K. & $\widehat{\delta}$ & $10 \mathrm{~m}$ & $P$ & $12(-)$ & $16(-)$ & 6,100 & 300 & - & + \\
\hline 9 & K.Y. & ㅇ & $2 \mathrm{~m}$ & A & $16(-$ & $16(-)$ & 4,160 & 296 & + & + \\
\hline 10 & T.T. & 官 & $9 \mathrm{~m}$ & $\mathrm{Lc}$ & $14(-$ & $16(-)$ & 4,900 & 390 & - & + \\
\hline 11 & T.A. & 우 & $8 \mathrm{~m}$ & $\mathrm{D}$ & $15( \pm)$ & $15( \pm)$ & 6,350 & 342 & - & + \\
\hline 12 & I. M. & 3 & $9 \mathrm{~m}$ & $\mathrm{D}$ & $12(-)$ & $14(-)$ & 6,300 & 259 & + & + \\
\hline 13 & M.S. & 우 & $5 \mathrm{~m}$ & $\mathrm{Br}$ & $16(-)$ & $14(-)$ & 3,500 & 300 & + & + \\
\hline 14 & T.A. & t & $2 \mathrm{~m}$ & $\mathrm{R}$ & $16(-)$ & $16(-)$ & 4,760 & 290 & + & + \\
\hline 15 & S. S. & 우 & $5 \mathrm{~m}$ & $B \mathbf{r}$ & $8(-3$ & $12(-)$ & 6,000 & 490 & + & $\dot{t}$ \\
\hline 16 & S.M. & 8 & $5 \mathrm{~m}$ & $\mathrm{Br}$ & 160 & $16(-)$ & 4,400 & 370 & + & + \\
\hline 17 & H.Y. & 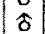 & $7 \mathrm{~m}$ & $\mathrm{R}$ & $16(-)$ & $14( \pm)$ & 5,120 & 280 & + & + \\
\hline 18 & M.K. & $\delta$ & $5 \mathrm{~m}$ & $\mathbf{D}+\mathbf{A}$ & $16(-)$ & 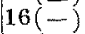 & 4,850 & 351 & \pm & + \\
\hline 19 & N.Y. & 今 & $2 \mathrm{~m}$ & $\mathrm{R}$ & $16(-)$ & $16(-)$ & 3,900 & 247 & + & - \\
\hline 20 & F.M. & 8 & $1 \mathrm{y} 3 \mathrm{~m}$ & $B$ & $14(-)$ & $16(-)$ & 5,480 & 322 & + & + \\
\hline 21 & T.0. & 우 & $3 \mathrm{~m}$ & $\mathrm{D}$ & $14(-$ & $14(-)$ & 4,760 & 293 & + & - \\
\hline 22 & D.K. & 客 & $6 \mathrm{~m}$ & $\mathrm{~A}$ & $8(-3)$ & $8(-)$ & 5,600 & 416 & + & + \\
\hline 23 & s. 0 . & ه & $48 \mathrm{~d}$ & $\mathbf{E}$ & $16(-)$ & $16(-)$ & 3,350 & 311 & + & - \\
\hline 24 & J.T. & $\delta$ & $3 \mathrm{~m}$ & $\mathbf{H}$ & $16(-)$ & $16(-)$ & 3,750 & 230 & $\ldots$ & + \\
\hline 25 & K.N. & क & $10 \mathrm{~m}$ & $\mathrm{Br}$ & $15(--)$ & $14(-)$ & 5,070 & 400 & + & - \\
\hline 26 & J. 0 . & 今 & $2 \mathrm{~m}$ & Le & $16(-)$ & $16(-)$ & 5,500 & 390 & + & - \\
\hline 27 & Y.I. & 무 & $6 \mathrm{~m}$ & $\mathrm{Bp}$ & $15(-)$ & $8(-)$ & 8,100 & 371 & 2 & + \\
\hline 28 & T. 0 . & 우 & $6 \mathrm{~m}$ & $\mathrm{Bp}$ & $16(-)$ & $16(-)$ & 5,250 & 810 & + & + \\
\hline 29 & A.I. & $\vec{\delta}$ & $9 \mathrm{~m}$ & $\mathrm{D}$ & $16(-)$ & $16(-)$ & 5,450 & 510 & + & + \\
\hline 30 & H.T. & 우 & $3 \mathrm{~m}$ & $\mathrm{D}$ & $16(-)$ & $16(-)$ & 6,250 & 553 & + & + \\
\hline 31 & S. W. & 우 & $4 \mathrm{~m}$ & $\mathrm{D}$ & $16(-)$ & $16(-)$ & 4,450 & 370 & + & + \\
\hline 32 & E.M. & $\delta$ & $4 \mathrm{~m}$ & B & $16(-)$ & $16(-)$ & 4,000 & 280 & + & - \\
\hline 33 & K.I. & t & $4 \mathrm{~m}$ & $\mathrm{H}$ & $16(-)$ & $15(-)$ & 3,330 & 263 & - & + \\
\hline 34 & F.M. & 숭 & $1 \mathrm{y} 3 \mathrm{~m}$ & $\mathrm{E}_{\mathrm{p}}$ & $8(-)$ & $14(-)$ & 4,850 & 368 & + & + \\
\hline 35 & T.0. & 우 & $6 \mathrm{~m}$ & $\mathrm{Br}$ & $16(-)$ & $16(-)$ & 5,100 & 730 & + & - \\
\hline 36 & T.S. & 우 & $4 \mathrm{~m}$ & $\mathrm{R}$ & $16(-)$ & $16(-)$ & 4,260 & 423 & - & + \\
\hline 37 & S.0. & + & $10 \mathrm{~m}$ & $\mathrm{~A}+\mathrm{Hi}$ & $16(-)$ & $16(-)$ & 4,880 & 360 & - & + \\
\hline
\end{tabular}

* Cf. foot note to Table 2.

878.7 thousands and my own result ${ }^{12)}$ was 299 thousands on an average. Thus I may well assume $250-350$ thousands as a normal platelet count.

From this standard, platelet count of normally Arakawa-positive group was quite ordinary, and when babies and/or their mothers had been taking vitamin B preparation, platelet count was only slightly 


\section{Table 8.}

Distribution of blood platelets and red cell count according to the intensity of A rak awa's reaction.

\begin{tabular}{c|c|c|c}
\hline Arak aw a's reaction & $\begin{array}{c}\text { Blood platelets } \\
\text { per cmm. }\end{array}$ & $\begin{array}{c}\text { Red cell connt } \\
\text { per cmm. }\end{array}$ & Cases \\
\hline Normally Arakawa-positive cases & 301,000 & $5,140,000$ & 23 \\
\hline $\begin{array}{c}\text { Cases with intermediate } \\
\text { Arak a w a's reaction }\end{array}$ & 384,000 & $5,130,000$ & 194 \\
\hline $\begin{array}{c}\text { Completely or almost completely } \\
\text { Arakawa-negative cases }\end{array}$ & 435,000 & $4,930,000$ & 128
\end{tabular}

TABLE 9.

Distribution of red cell count and blood platelet count according to the intensity of A rakawa's reaction and according as a vitamin $B$ preparation was taken or not.

\begin{tabular}{c|c|c|c|c}
\hline Ar a k a wa's reaction & $\begin{array}{c}\text { Administration } \\
\left(\begin{array}{c}+ \text { or no ad- } \\
\text { ministration (- } \\
\text { of vitamin B } \\
\text { preparation }\end{array}\right.\end{array}$ & $\begin{array}{c}\text { Blood platelets } \\
\text { per cmm. }\end{array}$ & $\begin{array}{c}\text { Red cell } \\
\text { count } \\
\text { per cmm. }\end{array}$ & Cases \\
\hline $\begin{array}{c}\text { Normally Arakawa- } \\
\text { positive cases }\end{array}$ & - & 317,000 & $5,140,000$ & 18 \\
\hline $\begin{array}{c}\text { Cases with intermediate } \\
\text { Arak a wa's reaction }\end{array}$ & + & 243,000 & $5,150,000$ & 5 \\
\hline $\begin{array}{c}\text { Completely or almost com- } \\
\text { pletely Arakawa-negative } \\
\text { cases }\end{array}$ & - & 397,000 & $5,100,000$ & 140 \\
\hline
\end{tabular}

over normal limit even in Arakawa-negative group (Cf. completely or almost completely Arakawa-negative cases in Table 9).

As to the literature concerning blood platelet count in the cases of infantile beriberi or B-avitaminosis, Kurimoto, Aoyama and Sakai ${ }^{18}$ stated simply an increase of thrombocytes without showing the actual number, and $\mathrm{Kohd \textrm {a } ^ { 1 9 }}$ said that platelet count was in the upper limit of physiologic variation. In the preceeding paper $\mathbf{I}^{12)}$

18) Y. Kurimoto, T. Aoy ama and T. Sakai, Zika Zassi, 1922, No. 263, 124.

19) S. Koh d a, Zika Zassi, 1935, No. 420, 607. 
Table 10.

Distribution of Arakawa-positive, intermediate and negative groups according to blood platelet count.

\begin{tabular}{|c|c|c|c|c|c|c|c|}
\hline \multirow{2}{*}{$\begin{array}{l}\text { Arakawa's } \\
\text { reaction }\end{array}$} & \multirow{2}{*}{$\begin{array}{l}\text { Administration } \\
(+) \text { or no ad- } \\
\text { ministration }(-) \\
\text { of vitamin } B \\
\text { preparation }\end{array}$} & \multicolumn{5}{|c|}{ Blood platelets per cmm. (thousands) } & \multirow{2}{*}{ Cases } \\
\hline & & $0-350$ & $351-450$ & $451-550$ & $551-650$ & Over 651 & \\
\hline \multirow{2}{*}{$\begin{array}{l}\text { Normally } \\
\text { Arakawa- } \\
\text { positive } \\
\text { cases }\end{array}$} & - & $\begin{array}{c}78 \% \\
\text { (14 eases) }\end{array}$ & $\begin{array}{c}11 \% \\
2 \text { cases }\end{array}$ & $\begin{array}{c}11 \% \\
2 \text { cases }\end{array}$ & - & - & 18 \\
\hline & + & $\left(\begin{array}{cc}100 & n \\
5 & n\end{array}\right)$ & - & 一 & - & - & 5 \\
\hline \multirow{2}{*}{$\begin{array}{l}\text { Cases with } \\
\text { intermediate } \\
\text { Arakawa's } \\
\text { reaction }\end{array}$} & - & $\left(\begin{array}{cc}27 & \\
(38 & \end{array}\right)$ & $\left\{\begin{array}{c}29 \% \\
(40 \text { cases })\end{array}\right.$ & $\begin{array}{c}31 \text { \% } \\
(43 \text { cases }\end{array}$ & $\left(\begin{array}{c}10 \% \\
14 \text { cases })\end{array}\right.$ & 5 cases & 140 \\
\hline & + & $\left(\begin{array}{cc}56 & n \\
(30 & n\end{array}\right)$ & $\left(\begin{array}{cc}28 & n \\
\left(15^{2}\right. & n\end{array}\right)$ & $\begin{array}{ll}13 & n\end{array}$ & $\left(2^{4} "\right.$ & - & 54 \\
\hline \multirow{2}{*}{$\begin{array}{c}\text { Completely } \\
\text { or almost } \\
\text { completely } \\
\text { Arakawa- } \\
\text { negative cases }\end{array}$} & - & $\begin{array}{c}28 \\
26\end{array}$ & )$\left(20^{22}\right.$ & $\begin{array}{c}30 " \\
27\end{array}$ & $9^{10} \quad n$ & $\begin{array}{c}10 \% \\
9 \text { cases }\end{array}$ & 91 \\
\hline & + & $\left(16^{43} \rrbracket\right)$ & $\left(16^{43} n\right.$ & 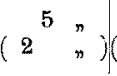 & $\begin{array}{cc}3 & n \\
1 & \text { case) }\end{array}$ & $2^{5}$ & 37 \\
\hline
\end{tabular}

TABLE 11.

The result shown in Table 10, simplified.

\begin{tabular}{|c|c|c|c|c|}
\hline \multirow{2}{*}{ Arakawa's reaction } & \multirow{2}{*}{$\begin{array}{c}\text { Administration } \\
(+) \text { or no ad- } \\
\text { ministration }(-) \\
\text { of vitamin } B \\
\text { preparations }\end{array}$} & \multicolumn{3}{|c|}{ Blood platelets per cmm. (thousands) } \\
\hline & & $0-350$ & $351-550$ & $551-$ \\
\hline \multirow{2}{*}{$\begin{array}{c}\text { Normally Arakawa- } \\
\text { positive cases }\end{array}$} & - & $78 \%$ & $22 \%$ & - \\
\hline & + & 100 & 一 & - \\
\hline \multirow{2}{*}{$\begin{array}{l}\text { Cases with intermediate } \\
\text { Arakawa's reaction }\end{array}$} & - & 27 & 49 & $14 \%$ \\
\hline & + & 56 & 41 & 4 \\
\hline \multirow{2}{*}{$\begin{array}{c}\text { Completely or almost com- } \\
\text { pletely Arakawa-uegative } \\
\text { cases }\end{array}$} & - & $28 \pi$ & 52 & 20 \\
\hline & + & 43 & 49 & 8 \\
\hline
\end{tabular}

pointed out platelet count was 400 thousands in the Arakawa-negative group and 477 thousands in avitaminosis $B$.

In my own result in this paper, thrombocyte count showed an increase, as above mentioned, according to the weakness of Ara- 
kawa's reaction. And so it can be said that sucklings fed on milk. negative to Arakawa's reaction were in a state of avitaminosis $B$ from the standpoint of thrombocytosis alone.

From the fact that in the same group of Arakawa's reaction platelets were smaller in the group of infants and/or their mothers taking vitamin $B$, it is evident that there was a close relation between vitamin $\mathrm{B}$ and blood platelet count, as was also pointed out by Kohda. ${ }^{19}$

In the Arakawa-negative group anemia was seen in a slight degree. This agrees with the finding of Takas ${ }^{20)}$ ' and $\mathrm{Ku}$ rimoto, A oyama and Sakai, ${ }^{18)}$ but is opposed to that of Fukushima ${ }^{21)}$ who reported an increase of red cell count in a slight degree.

I should have experimented on "healthy" infants with no complaint, but it is very difficult to collect a large number of such materials. In the present paper I excepted serious cases which might affect the platelet count to a high degree. But I did not expect to see such a striking difference of platelet count between Arakawa-positive and Arakawa-negative groups as obtained in the present investigation. I expected to be able to see such an inclination only in these sick infants.

It is noteworthy that blood platelet count showed an increase as Arakawa's reaction became weaker, in spite of the possibility, that the platelets of these sick infants might have been influenced by the type, severity, and stage of their disease.

In the near future I will report again of the relation between blood platelet count and A rakawa's reaction in the course of such diseases in which platelet count will not be influenced by the disease itself.

\section{Summary,}

1. Blood platelet count of infants showed an increase in the case of mothers with negative Arakawa's reaction, being 435, 384 and 301 thousand thrombocytes in the Arakawa-negative group, in the group of intermediate Arakawa's reaction, and in the normally Arakawa-positive group réspectively.

2. Red cell count was 5,140 and 5,130 thousand cells in the strongly Arakawa-positive group and in the group of intermediate A rakawa's reaction, while a slight anemia was seen in the Arakawa-

20) K. Takas u, Tokio Igaku-kwai Zassi, 1903, 17, 395.

21) T. Fukushima, Nynji-gaku Zassi, 1932, 12, 371. 
negative group showing 4,930 thousand on an average.

3. In the cases in which infants and their mothers had not been taking vitamin B preparations, platelet count was within normal (317 thousands) in the Arakawa-positive group, but an increase of platelet count (397 thousands) was seen in the cases with intermediate Arakawa's reaction, and the maximal average count (458 thousands) occurred in the Arakawa-negative group.

4. In the cases in which infants and/or their mothers had been taking vitamin $B$ preparations, blood platelet count was a moderate surplus over the normal limit even in the Arakawa-negative group.

\section{Concluisions.}

An increase of blood platelet count was seen in infants fed on milk negative to A rakaw a's reaction. When infants and/or their mothers had been taking a vitamin B preparation, the platelet count was only moderately over the normal limit even in the Arakawa-negative group. There is thus a close relation between blood platelet count of infants and Arakawa's reaction of their mothers.

It is to be added here that infants of the completely Arakawanegative mothers have a reduced red cell count, in spite of the fact that those of mothers with intermediate A rakaw a's reaction are of almost the same red cell count as those of Arakawa-positive mothers. 